ARTÍCULO ORIGINAL

\title{
ANÁLISIS CLÎNICO-EPIDEMIOLÓGICO DE LAS SUBFRACCIONES HDL2 Y HDL3 EN ADULTOS DE LA CIUDAD DE MARACAIBO, VENEZUELA
}

\author{
Sergia Linares $\mathbb{Q}^{1, a, b}$, Valmore Bermúdez $\mathbb{1}^{2, b}$, Juan Salazar $\mathbb{1}^{1, c, d}$, Manuel Nava $\mathbb{1}^{1, e}$,

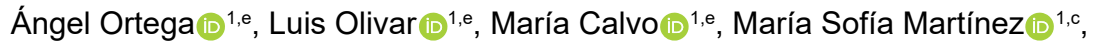

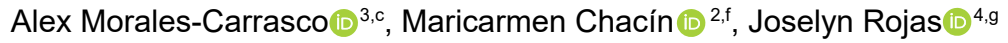 \\ 1 Centro de Investigaciones Endocrino-Metabólicas «Dr. Félix Gómez», Escuela de Medicina, Universidad del Zulia, \\ Maracaibo, Venezuela. \\ ${ }^{2}$ Universidad Simón Bolívar, Facultad de Ciencias de la Salud, Barranquilla, Colombia. \\ 3 Universidad Técnica de Ambato, Ambato, Ecuador. \\ ${ }^{4}$ Pulmonary and Critical Care Medicine Department, Brigham and Women's Hospital, Harvard Medical School, Boston, \\ Massachusetts, Estados Unidos. \\ ${ }^{\text {a }}$ Magíster en Nutrición; ${ }^{\mathrm{b}}$ magíster en Metabolismo Humano, doctor en Ciencias Médicas; ${ }^{\mathrm{c}}$ médico cirujano; ${ }^{\mathrm{d}}$ especialista \\ en Medicina Interna; ${ }^{\mathrm{e}}$ estudiante de Medicina Humana; ${ }^{\mathrm{f}}$ magíster en Dermatología; ${ }^{\mathrm{g}}$ magíster en Inmunología.
}

\section{RESUMEN}

Objetivo: Realizar un análisis clínico-epidemiológico de las subfracciones de colesterol unido a lipoproteinas de alta densidad (HDL-C, por sus siglas en inglés) en adultos de la ciudad de Maracaibo, Venezuela. Materiales y métodos: Se realizó un estudio descriptivo y transversal de la base de datos del Estudio de Prevalencia de Síndrome Metabólico de Maracaibo, que incluyó 359 individuos de ambos sexos, mayores de 18 años, a quienes se les determinó la concentración sérica de HDL3 y HDL2, así como el índice HDL2/ HDL3; evaluando sus niveles según características sociodemográficas, clínicas y bioquímicas. Resultados: La edad promedio de la población era $39,4 \pm 15,2$ años, y $51,5 \%$ era de sexo femenino. Solo se observaron diferencias en los niveles de HDL-C en aquellos sujetos con HDL-C bajas. Las mujeres con hipertriacilgliceridemia mostraron concentraciones séricas de HDL3 y HDL2 significativamente menores con respecto a aquellas con triacilglicéridos normales $(\mathrm{p}=0,033)$; asimismo, se encontró una concentración menor de HDL3 y relación HDL2/HDL3 en aquellas con proteína C reactiva ultrasensible (PCR-us) elevada $(\mathrm{p}<0,001)$. En hombres, se evidenció una concentración significativamente menor de HDL2 en aquellos con algún grado de hipertensión arterial $(\mathrm{p}=0,031)$, insulinorresistencia $(\mathrm{p}=0,050)$ y síndrome metabólico $(\mathrm{p}=0,003)$; mientras que aquellos con PCR-us elevada mostraron una menor concentración de HDL3 $(p=0,011)$. Conclusión: Las subfracciones de HDL-C muestran un comportamiento clínico epidemiológico variable en adultos de la población de Maracaibo, con promedios más bajos en los hombres, diferencias en los niveles únicamente en aquellos con HDL-C bajas, y sin predominio de alguna subclase según las características sociodemográficas, clínicas y bioquímicas.

Palabras clave: Lipoproteínas HDL3; Lipoproteínas HDL2; HDL-Colesterol; Factores de Riesgo; Epidemiología (fuente: DeCS BIREME).

Citar como: Linares S, Bermúdez V, Salazar J, Nava M, Ortega A, Olivar L, et al. Análisis clínico-epidemiológico de las subfracciones HDL2 y HDL3 en adultos de la ciudad de Maracaibo, Venezuela. Rev Peru Med Exp Salud Publica. 2020;37(3):412-22. doi: https://doi.org/10.17843/ rpmesp.2020.373.4787.

\footnotetext{
Correspondencia: Juan Salazar; Centro de Investigaciones Endocrino-Metabólicas «Dr. Félix Gómez», Escuela de Medicina, Universidad del Zulia. Maracaibo 4004, Venezuela; juanjsv18@hotmail.com
}

\section{CLINICAL-EPIDEMIOLOGICAL ANALYSIS OF HDL2 AND HDL3 SUBFRACTIONS IN ADULTS FROM MARACAIBO CITY, VENEZUELA}

\section{ABSTRACT}

Objective: To carry out a clinical-epidemiological analysis of high-density lipoprotein cholesterol subfractions (HDL-C) in adults from Maracaibo, Venezuela. Materials and methods: A descriptive and cross-sectional study of the database from the Metbolic Syndrome Prevalence in Maracaibo Study was carried out. HDL3 and HDL2 serum concentration, as well as the HDL2/HDL3 ratio, were determined in 359 individuals of both sexes, over 18 years of age. Values obtained were evaluated according to sociodemographic, clinical and biochemical characteristics. Results: Mean population age was $39.4 \pm 15.2$ years, and 51.5\% were female. Differences in HDL-C subfraction levels were only observed in those subjects with low HDL-C levels. Women with hypertriglyceridemia showed significantly lower serum HDL3 and HDL2 concentrations than those with normal triglycerides ( $\mathrm{p}=0.033$ ), as well as a lower HDL3 level and HDL2 / HDL3 ratio in those with higher levels of ultra-sensitive C-reactive protein (us-CRP) $(\mathrm{p}<0.001)$. A significantly lower concentration of HDL2 was observed in men with some degree of hypertension $(p=0.031)$, insulin resistance $(p=0.050)$ and metabolic syndrome $(\mathrm{p}=0.003)$; while those with elevated us-CRP showed a lower concentration of HDL3 ( $\mathrm{p}=0.011)$. Conclusion: HDL-C subfractions show varying clinical-epidemiological behavior in adults from Maracaibo. Lower serum levels are observed in men, differences only in those with low HDL-C; and no predominance of any subclass was observed according to sociodemographic, clinical and biochemical characteristics.

Keywords: Lipoproteins, HDL3; Lipoproteins, HDL2; Cholesterol, HDL; Risk Factors; Epidemiology (source: MeSH NLM). 


\section{INTRODUCCIÓN}

Las lipoproteínas de alta densidad (HDL, por sus siglas en inglés) son complejos macromoleculares, seudomicelares, cuya función más conocida es transportar el colesterol desde los tejidos periféricos hacia el hígado para su metabolismo y excreción, proceso conocido como transporte reverso de colesterol (TRC). Estas lipoproteínas tienen, además, propiedades antitrombóticas, antiinflamatorias, vasodilatadoras, inmunosupresoras y antioxidantes ${ }^{(1)}$. Lo anterior ha llevado a considerar durante años a las HDL como moléculas protectoras para la enfermedad cardiovascular (ECV), calificada en la actualidad un problema de salud pública global por ser la primera causa de muerte en todo el mundo, ésta causó 17,8 millones de defunciones en el $2017^{(2)}$. En la población de Maracaibo, se ha observado previamente una alta prevalencia de bajos niveles de colesterol transportado por lipoproteínas de alta densidad (HDL-C) ${ }^{(3)}$; sin embargo, el riesgo coronario parece ser menor que en otras poblaciones con frecuencia más baja de esta alteración lipídica ${ }^{(4)}$.

Aunque existe evidencia epidemiológica de que HDL-C es un predictor independiente de la ECV, los ensayos clínicos farmacológicos enfocados en su mejoría cuantitativa no han demostrado beneficios en términos de reducción de eventos o mortalidad ${ }^{(5)}$. Este fenómeno paradójico ha sido justificado ante la heterogeneidad y complejidad que muestran estas moléculas, cuya funcionalidad es la característica fundamental en su papel como "protector» cardiovascular ${ }^{(6)}$. En este sentido, parece existir una relación estrecha entre la función y las subfracciones o subespecies de HDL-C ${ }^{(7)}$.

De Lalla et al. ${ }^{(8)}$ describieron por primera vez la distribución de las subfracciones de HDL-C e identificaron dos subclases: la HDL2, la menos densa (1,063-1,125 g/mL) y rica en lípidos, conocida como HDL2; y la HDL3, la más densa $(1,125-1,21 \mathrm{~g} / \mathrm{mL})$ y relativamente rica en proteínas. HDL3 y HDL2 parecen poseer funciones diferentes, las HDL3 tienen la capacidad de recibir el colesterol libre desde el endotelio o macrófagos, mediante el transportador dependiente de ATP-binding cassette transporter A-1 (ABCA1), aumentando su tamaño con disminución de su densidad, transformándose así en HDL2, las cuales, son responsables del TRC al unirse a los receptores SR-B1, por lo cual, el papel de cada subfracción en la salud cardiometabólica podría ser diferente ${ }^{(9)}$.

Los hallazgos epidemiológicos relacionados con las subfracciones son diversos y controversiales, razón por la cual, actualmente ninguna guía internacional de diagnóstico y manejo de dislipidemia recomienda su evaluación rutinaria en los pacientes con riesgo cardiovascular. No obstante, diversos reportes plantean el potencial papel que tendrían estas subfracciones como marcadores de funcionalidad de las $\mathrm{HDL}$, y el efecto modulador que tendrían los fármacos sobre la $\mathrm{ECV}^{(7,10)}$.

\section{MENSAJES CLAVE}

\begin{abstract}
Motivación para realizar el estudio: Se desconoce el comportamiento epidemiológico de las subfracciones de HDL-C y su relación con otras alteraciones metabólicas en poblaciones latinoamericanas, especialmente de Venezuela.
\end{abstract}

Principales hallazgos: El promedio de las subfracciones de HDL-C fue más bajo en hombres, las diferencias en los valores se evidenciaron únicamente en sujetos con HDL-C bajas. Las subfraccciones mostraron niveles más bajos en hombres y mujeres con alteraciones clínicas y metabólicas, con un comportamiento variable.

Implicancias: Las subfracciones de HDL-C representan parámetros de laboratorio de potencial utilidad en individuos con HDL-C bajas, su determinación en este contexto permitiría la identificación temprana de sujetos con factores de riesgo, especialmente en aquellos con riesgo cardiovascular intermedio o bajo.

En Latinoamérica, especialmente en Venezuela, pese a que las HDL-C bajas son una dislipidemia de alta frecuencia, son escasos o inexistentes los reportes que describen el comportamiento de las subfracciones de HDL o su relación con otros factores de riesgo cardiovascular. Por lo cual, el objetivo del presente estudio fue realizar un análisis clínico-epidemiológico de las subfracciones HDL2 y HDL3 en una población adulta de la ciudad de Maracaibo, Venezuela.

\section{MATERIALES Y MÉTODOS}

\section{Población y muestra}

Los datos de esta investigación provienen del «Estudio de Prevalencia de Síndrome Metabólico de la Ciudad de Maracaibo (EPSMM)» de tipo transversal, descriptivo, analítico y con muestreo de tipo aleatorio multietápico, para el cual se incluyeron 2230 adultos de ambos sexos, residentes en Maracaibo, diseñado para identificar y evaluar factores de riesgo del síndrome metabólico y la enfermedad cardiovascular. La muestra del estudio principal se calculó sobre la base de las estimaciones censales del Instituto Nacional de Estadística para la ciudad de Maracaibo del 2007, con una población de 1428043 habitantes mayores de 18 años, la muestra calculada fue de 2230 individuos, seleccionados aleatoria y estratificadamente en las 18 parroquias que conforman la ciudad. El protocolo fue descrito anteriormente ${ }^{(11)}$.

Para el estudio de las subfracciones de HDL, se realizó un subanálisis con los datos del EPSMM y, ante la falta de disponibilidad de recursos materiales para determinar 
las subfracciones en todos los individuos con HDL-C bajas, se seleccionaron 359 sujetos de manera aleatoria de la base de datos mediante la herramienta de números aleatorios del programa SPSS, manteniendo una distribución equitativa por sexo, grupos etarios y presencia de HDL-C bajas. A esta submuestra de individuos, las determinaciones de subfracciones de HDL fueron recolectadas y procesadas como parte del estudio en 2012.

\section{Procedimiento}

\section{Evaluación de los sujetos}

Personal previamente capacitado completó la historia clínica de los participantes, con información relacionada con la edad, sexo, raza, estado laboral, estrato socioeconómico (estrato I [clase alta], estrato II [clase media-alta], estrato III [clase media], estrato IV [clase obrera] y estrato V [pobreza extrema]); nivel educativo (hasta primaria: aquellos que no tenían algún conocimiento de lectoescritura o aquellos que completaron la educación primaria; secundaria: todos aquellos que terminaron la educación secundaria; y superior: todos aquellos que culminaron algún grado de educación superior); antecedentes patológicos familiares y personales de enfermedades endocrino-metabólicas o cardiovasculares.

En cuanto a los hábitos psicobiológicos, el tabaquismo se categorizó en 1) fumadores actuales, 2) no fumadores, y 3) exfumadores (al menos un año de haber dejado el hábito tabáquico). El consumo de alcohol se definió como la ingesta de $>1 \mathrm{~g} /$ día. Los niveles de actividad física fueron determinados usando la versión larga del Cuestionario Internacional de Actividad Física (IPAQ, por sus siglas en inglés) y se clasificó en dos grandes grupos: 1) aquellos individuos con MET's=0 (ninguna) y 2) aquellos con MET'S $>0$ (algún grado de actividad física). Este último grupo fue dividido posteriormente en quintiles obteniendo la siguiente clasificación: Q1 o muy baja (hombres: <296,9; mujeres: <230,9), Q2 o baja (hombres: 297,0-791,9; mujeres: 231,0-445,499), Q3 o moderado (hombres: 792,0-1532,3; mujeres: 445,5-742,4), Q4 o alta (hombres: 1532,4- 2879,9; mujeres: 742,5-1798,4), Q5 o muy alta (hombres: $\geq 2880,0$; mujeres: $\geq 1798,5$ ).

\section{Evaluación clínica}

Se usó un tensiómetro calibrado y validado para medir la tensión arterial de los participantes. Se emplearon los criterios planteados por el séptimo reporte del Comité Nacional Conjunto en Prevención, Detección, Evaluación y Tratamiento de la hipertensión arterial (JNC-7, por sus siglas en inglés) para clasificar a la población en normotensos, prehipertensos e hipertensos ${ }^{(12)}$.

Además, se realizó una evaluación antropométrica, para determinar el peso se usó una balanza digital (Tanita, TBF-310 GS Body Composition Analyzer, Tokio-Japón), y para la talla, un tallímetro calibrado. El índice de masa corporal (IMC) se calculó mediante la fórmula de Quetelec [peso/talla ${ }^{2}$ y se clasificó a los sujetos según los criterios de la Organización Mundial de la Salud (OMS): bajo peso $\left(<18,5 \mathrm{~kg} / \mathrm{m}^{2}\right)$, normopeso $\left(18,5-24,9 \mathrm{~kg} / \mathrm{m}^{2}\right)$, sobrepeso $\left(25,0-29,9 \mathrm{~kg} / \mathrm{m}^{2}\right)$, obesidad $\left(\geq 30,0 \mathrm{~kg} / \mathrm{m}^{2}\right)^{(13)}$. Se midió la circunferencia abdominal mediante una cinta métrica graduada en centímetros y milímetros, tomando como referencia anatómica un punto equidistante entre el reborde costal y la cresta iliaca antero-superior, los valores utilizados para definir obesidad abdominal fueron $\geq 90 \mathrm{~cm}$ para hombres $\mathrm{y} \geq 80 \mathrm{~cm}$ para mujeres según los criterios del consenso IDF/NHLBIAHA/WHF/IAS/ IASO $^{(14)}$, así como el diagnóstico del síndrome metabólico (SM).

\section{Análisis de laboratorio}

Luego de ocho horas de ayuno, se calcularon los niveles séricos de glucosa, colesterol total y triacilglicéridos empleando kits comerciales enzimáticos-colorimétricos (Human Gesellshoft Biochemica and Diagnostica $\mathrm{MBH}$, Hessen, Alemania) y un equipo computarizado especializado; los niveles de LDL-C se calcularon aplicando la fórmula de Friedewald. Los niveles séricos de proteína $C$ reactiva ultrasensible (PCR-us) se cuantificaron mediante ensayos inmunoturbodimétricos (Human Gesellshoft Biochemica and Diagnostica MBH, Hessen, Alemania), utilizándose como punto de corte un valor $\geq 0,765 \mathrm{mg} / \mathrm{L}$ para definir niveles elevados ${ }^{(15)}$. La concentración basal de insulina se determinó mediante un kit comercial basado en el método de ELISA (DRG Internacional, Inc., EUA, Nueva Jersey), con un límite de detección $<1 \mathrm{mU} / \mathrm{L}$. Por su parte, los valores de lipoproteína (Lp[a]) se estimaron a través del método turbidimétrico de látex (Human Gesellschaft für Biochemica and Diagnostica, Hessen, Alemania). El punto de corte para considerar valores elevados de $\mathrm{Lp}$ (a) fue $\geq 30 \mathrm{mg} / \mathrm{dL}^{(16)}$. Se calcularon los valores de HOMA2-IR con el software (HOMA-Calculator v2.2.3) del Oxford Centre for Diabetes Endocrinology and Metabolism, disponible en http://www.dtu.ox.ac. uk/homacalculator/index.php, para definir insulinorresistencia (IR). El punto de corte usado para HOMA2-IR fue 2,0, establecido previamente para la población de estudio ${ }^{(17)}$.

Las HDL se aislaron según el método de ultracentrifugación secuencial, se dializaron contra una solución amortiguadora Tris $0,09 \mathrm{M}$-ácido bórico 0,08M- EDTANa $23 \mathrm{Mm}, \mathrm{pH}=8,35$ (TBE). Seguidamente, se realizó una electroforesis en gradiente de gel de poliacrilamida en condiciones no desnaturalizantes, donde se usaron marcadores proteicos calibrados en diámetro molecular (tiroglobulina 17,0 nm; ferritina 12,2 nm; catalasa $10,4 \mathrm{~nm}$; lactato deshidrogenasa $8,1 \mathrm{~nm}$ y albúmina $7,1 \mathrm{~nm}$; Amersham Pharmacia Biotech, Buckimghamshire, Reino Unido). La proteína total fue cuantificada mediante una modificación del método de Lowry, las bandas de proteína HDL se tiñeron con azul de Coomassie R-250 y el diámetro promedio se determinó por densitometría óptica. 
La estimación del contenido relativo de apolipoproteínas se realizó mediante una electroforesis de las HDL en gradiente de poliacrilamida 4-21\%, tiñéndolas con azul de Coomassie R-250 y el gel se analizó por densitometría óptica. Los resultados se obtuvieron como el porcentaje que representa el área bajo la curva de cada apolipoproteína según la sumatoria de las áreas de las apolipoproteínas-HDL. Las subfracciones de HDL se aislaron con el kit Quantolip ${ }^{\circledR}$ HDL (HDL2/HDL3) (Technoclone, Viena, Austria). Asimismo, se calculó el índice HDL2/ HDL3 para evaluar la relación entre estas variables.

\section{Análisis estadístico}

Las variables nominales y ordinales se presentan como frecuencias absolutas y relativas. Se determinó la asociación entre variables cualitativas usando la prueba Chi-cuadrado y se evaluó la normalidad de las variables cuantitativas con la prueba $\mathrm{Z}$ de Kolmogorov-Smirnov. Estas variables se presentaron por medias \pm desviación estándar (DE). Para comparar promedios entre grupos se utilizó la prueba t-Student para comparar dos grupos y un análisis de varianza (ANOVA) de un factor para más de dos grupos. Se consideró significativo un valor de $\mathrm{p}<0,05$; el análisis estadístico se realizó con el programa SPSS 20,0 (IBM, EUA).

\section{Aspectos éticos}

El comité de ética del Centro de Investigaciones Endocrino-Metabólicas (CIEM) «Dr. Félix Gómez» de la Universidad del Zulia, Venezuela, aprobó el estudio y autorizó el uso de la base de datos para este subanálisis. Todos los participantes firmaron un consentimiento informado antes de cualquier intervención, interrogación y examen físico.

\section{RESULTADOS}

\section{Características de la población}

En la Tabla 1 se muestran las características generales de la muestra estudiada, constituida por 359 individuos, de los cuales un $51,5 \%(n=185)$ eran de sexo femenino. El promedio de edad en la población general fue 39,4 \pm 15,2 años. Con respecto al comportamiento de las HDL-C según sexo (Figura 1), se evidenció que la concentración de esta lipoproteína fue significativamente mayor en las mujeres respecto a los hombres $(47,2 \pm 13,4 \mathrm{mg} / \mathrm{dL}$ vs. $40,1 \pm 9,4$ $\mathrm{mg} / \mathrm{dL} ; \mathrm{p}<0,001)$. Asimismo, se encontraron diferencias estadísticamente significativas en la concentración de HDL3 (mujeres: $28,9 \pm 11,9 \mathrm{mg} / \mathrm{dL}$ vs. hombres: $25,1 \pm 8,6 \mathrm{mg}$ / $\mathrm{dL} ; \mathrm{p}=0,004)$ y de HDL2 (mujeres: $18,2 \pm 9,2 \mathrm{mg} / \mathrm{dL}$ vs. hombres: $15,0 \pm 8,3 \mathrm{mg} / \mathrm{dL} ; \mathrm{p}=0,001)$.

\section{Percentiles de las subfracciones de HDL-C}

En la Tabla 2 se muestra la distribución por percentiles de las subfracciones de HDL-C según sexo. Para mujeres, el promedio de HDL3 fue 26,7 mg/dL $\left(\mathrm{P}_{25}-\mathrm{P}_{75}: 19,9-36,9\right)$; de

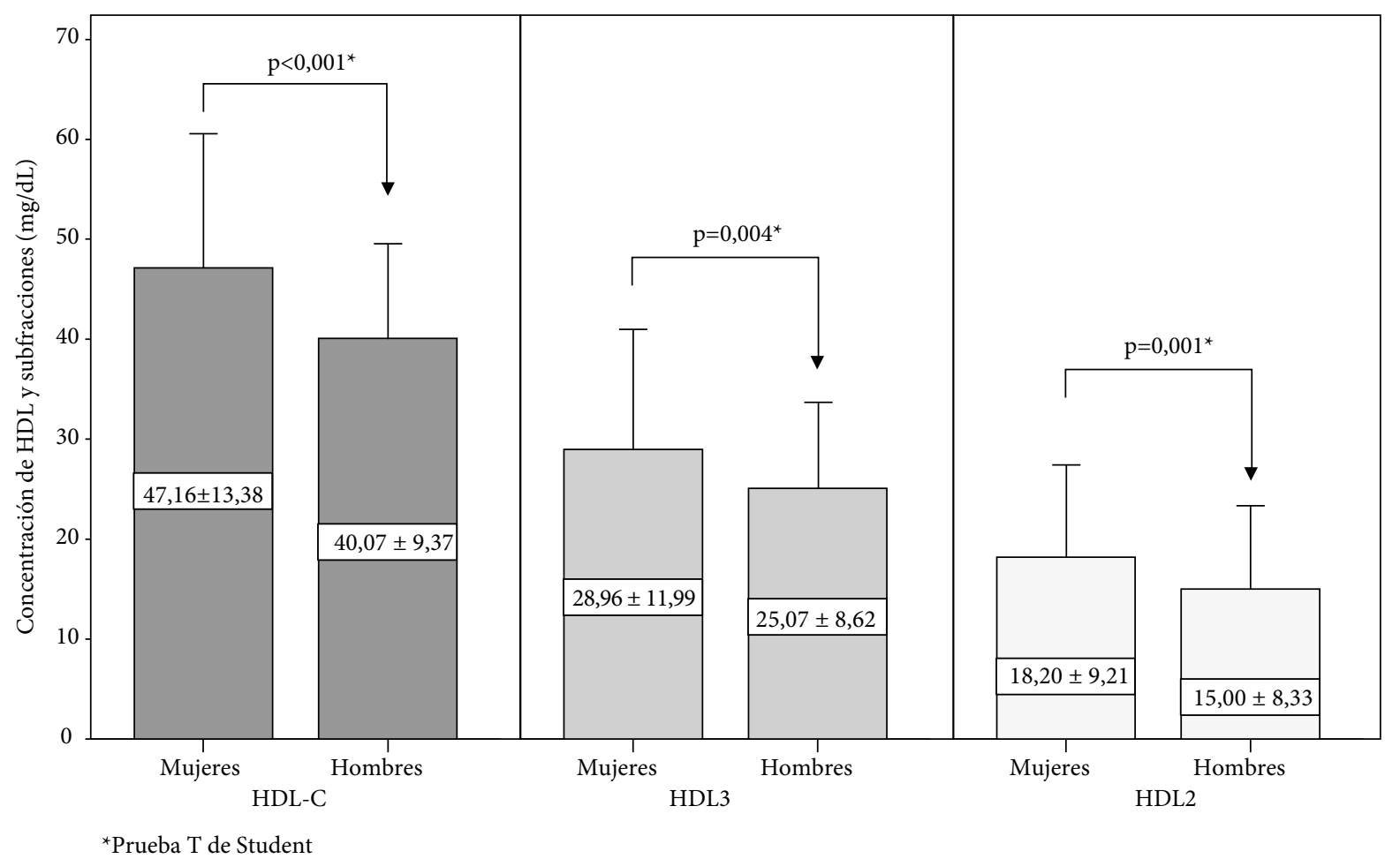

Figura 1. Comportamiento epidemiológico de la concentración de HDL-C y subfracciones según sexo. 
Tabla 1. Características generales de la población estudiada

\begin{tabular}{|c|c|c|c|c|c|c|}
\hline \multirow{3}{*}{ Características } & \multirow{2}{*}{\multicolumn{2}{|c|}{$\begin{array}{l}\text { Mujeres } \\
(n=185)\end{array}$}} & \multirow{2}{*}{\multicolumn{2}{|c|}{$\begin{array}{l}\text { Hombres } \\
(n=174)\end{array}$}} & \multirow{2}{*}{\multicolumn{2}{|c|}{$\frac{\text { Total }}{(n=359)}$}} \\
\hline & & & & & & \\
\hline & $\mathbf{n}$ & $\%$ & $\mathbf{n}$ & $\%$ & $\mathbf{n}$ & $\%$ \\
\hline \multicolumn{7}{|l|}{ Grupo etario (años) } \\
\hline$<30$ & 54 & 29,2 & 67 & 38,5 & 121 & 33,7 \\
\hline $30-49$ & 71 & 38,4 & 63 & 36,2 & 134 & 37,3 \\
\hline$\geq 50$ & 60 & 32,4 & 44 & 25,3 & 104 & 29,0 \\
\hline \multicolumn{7}{|l|}{ Estado civil } \\
\hline Soltero & 95 & 51,4 & 82 & 47,1 & 177 & 49,3 \\
\hline Casado & 90 & 48,6 & 92 & 52,9 & 182 & 50,7 \\
\hline \multicolumn{7}{|l|}{ Condición laboral } \\
\hline Empleado & 81 & 43,8 & 135 & 77,6 & 216 & 60,2 \\
\hline Desempleado & 104 & 56,2 & 39 & 22,4 & 143 & 39,8 \\
\hline \multicolumn{7}{|l|}{ Nivel educativo } \\
\hline Hasta primaria & 38 & 20,5 & 20 & 11,5 & 58 & 16,2 \\
\hline Secundaria & 78 & 42,2 & 88 & 50,6 & 166 & 46,2 \\
\hline Superior & 69 & 37,3 & 66 & 37,9 & 135 & 37,6 \\
\hline \multicolumn{7}{|l|}{ Estrato socioeconómico } \\
\hline I-II & 34 & 18,4 & 35 & 20,1 & 69 & 19,2 \\
\hline III & 87 & 47,0 & 77 & 44,3 & 164 & 45,7 \\
\hline IV-V & 64 & 34,6 & 62 & 35,6 & 126 & 35,1 \\
\hline \multicolumn{7}{|l|}{ Raza } \\
\hline Mestiza & 141 & 76,6 & 134 & 77,0 & 275 & 76,8 \\
\hline Blanco hispánica & 24 & 13,0 & 26 & 14,9 & 50 & 14,0 \\
\hline Afrovenezolana & 3 & 1,6 & 5 & 2,9 & 8 & 2,2 \\
\hline Indígena americano & 16 & 8,7 & 9 & 5,2 & 25 & 7,0 \\
\hline \multicolumn{7}{|l|}{ Consumo de alcohol ${ }^{*}$} \\
\hline Sí & 33 & 17,8 & 100 & 57,5 & 133 & 37,0 \\
\hline No & 152 & 82,2 & 74 & 42,5 & 226 & 63,0 \\
\hline \multicolumn{7}{|l|}{ Hábito tabáquico } \\
\hline No & 153 & 82,7 & 111 & 63,8 & 264 & 73,5 \\
\hline Fumador & 14 & 7,6 & 26 & 14,9 & 40 & 11,1 \\
\hline Exfumador & 18 & 9,7 & 37 & 21,3 & 55 & 15,3 \\
\hline \multicolumn{7}{|l|}{ AF en esfera ocio } \\
\hline Ninguna & 127 & 68,6 & 82 & 47,1 & 209 & 58,2 \\
\hline Muy baja & 7 & 3,8 & 19 & 10,9 & 26 & 7,2 \\
\hline Baja & 19 & 10,3 & 15 & 8,6 & 34 & 9,5 \\
\hline Moderada & 11 & 5,9 & 21 & 12,1 & 32 & 8,9 \\
\hline Alta & 10 & 5,4 & 14 & 8,0 & 24 & 6,7 \\
\hline Muy alta & 11 & 5,9 & 23 & 13,2 & 34 & 9,5 \\
\hline \multicolumn{7}{|l|}{ HDL-C bajas } \\
\hline No & 86 & 46,5 & 84 & 48,3 & 170 & 47,4 \\
\hline Sí & 99 & 53,5 & 90 & 51,7 & 189 & 52,6 \\
\hline
\end{tabular}

AF: actividad física.

${ }^{\star}$ Consumidor $>1 \mathrm{~g} /$ día 
Tabla 2. Percentiles de las subfracciones de HDL en la población estudiada

\begin{tabular}{|c|c|c|c|c|c|c|}
\hline Variable & p25 & p33,3 & Mediana & p66,6 & p75 & p95 \\
\hline \multicolumn{7}{|l|}{ Mujeres $(\mathrm{n}=185)$} \\
\hline HDL3 (mg/dL & 19,9 & 22,7 & 26,7 & 33,1 & 36,9 & 49,8 \\
\hline HDL2 (mg/dL) & 10,7 & 13,0 & 17,6 & 20,8 & 24,6 & 36,0 \\
\hline HDL2/HDL3 & 0,35 & 0,43 & 0,66 & 0,86 & 1,02 & 1,89 \\
\hline \multicolumn{7}{|l|}{ Hombres $(\mathrm{n}=174)$} \\
\hline HDL3 (mg/dL) & 18,9 & 21,0 & 24,8 & 28,9 & 30,5 & 42,2 \\
\hline HDL2 (mg/dL) & 9,2 & 10,9 & 13,8 & 16,1 & 18,1 & 33,6 \\
\hline HDL2/HDL3 & 0,35 & 0,41 & 0,56 & 0,74 & 0,83 & 2,47 \\
\hline
\end{tabular}

p: percentil

HDL2 fue 17,6 mg/dL $\left(\mathrm{P}_{25}-\mathrm{P}_{75}: 10,7-24,6\right)$; y para la relación HDL2/HDL3 fue 0,66 ( $\left.\mathrm{P}_{25}-\mathrm{P}_{75}: 0,35-1,02\right)$. Mientras que en hombres, el promedio de HDL3 fue $24,8 \mathrm{mg} / \mathrm{dL}\left(\mathrm{P}_{25}-\mathrm{P}_{75}\right.$ : 18,9-30,5); de HDL2 fue 13,8 mg/dL $\left(\mathrm{P}_{25}-\mathrm{P}_{75}: 9,2-18,1\right)$; $\mathrm{y}$ para la relación HDL2/HDL3: 0,56 $\left(\mathrm{P}_{25}-\mathrm{P}_{75}: 0,35-0,83\right)$.

Subfracciones de HDL-C según características sociodemográficas y hábitos

En la Tabla 3 se observa el comportamiento de las HDL2 y HDL3 en individuos con HDL-C bajas según variables sociodemográficas. Las mujeres de estratos socioeconómicos IV-V presentaron valores significativamente menores de HDL3 comparados con los estratos socioeconómicos superiores ( $\mathrm{p}<0,001)$. Asimismo, en los estratos IV-V presentaron una mayor relación HDL2/HDL3 con respecto al estrato I-II $(0,9 \pm 0,5$ vs. $0,7 \pm 0,6$, respectivamente; $\mathrm{p}=0,012$ ).

En relación a la raza, se evidenció una mayor concentración de HDL2 y HDL2/HDL3 en los indígenas americanos en comparación con los individuos blanco-hispánicos (HDL2: 17,8 $\pm 5,0 \mathrm{mg} / \mathrm{dL}$ vs. $9,8 \pm 4,2 \mathrm{mg} / \mathrm{dL} ; \mathrm{p}=0,040 \mathrm{y}$ HDL2/HDL3: $1,1 \pm$ 0,45 vs. $0,5 \pm 0,3 ; \mathrm{p}=0,007$, respectivamente). Por el contrario, en hombres con HDL-C bajas no se encontraron diferencias significativas en los promedios de las subfracciones con respecto a las variables sociodemográficas.

En cuanto a los hábitos psicobiológicos, en mujeres se observó que las no fumadoras presentaron mayores niveles de HDL3 con respecto a las exfumadoras $(22,4 \pm 6,6 \mathrm{mg} / \mathrm{dL}$ vs. $17,1 \pm 7,6 \mathrm{mg} /$ $\mathrm{dL}$, respectivamente, $\mathrm{p}=0,034)$. Por el contrario, los hombres con HDL-C bajas solo mostraron diferencias significativas entre el nivel de actividad física en la esfera de ocio y el índice HDL2/ HDL3 ( $p=0,038)$, sin observarse diferencias importantes entre los valores del índice en cada rango de actividad.

Subfracciones de HDL-C según características clínico-metabólicas Con respecto a las alteraciones clínico-metabólicas, se observó (Tabla 4) que las mujeres con HDL-C bajas e hipertriacilgliceri- demia mostraron concentraciones séricas de HDL3 y HDL2 significativamente menores en comparación a aquellas con tricilglicéridos (TAG) normales $(\mathrm{p}=0,033$ y $\mathrm{p}=0,034$, respectivamente). Se han encontrado hallazgos similares en mujeres con PCR-us elevada, donde se observó una concentración menor de HDL3 y una relación HDL2/HDL3, con respecto a aquellas con PCR-us normal ( $\mathrm{p}<0,001$ y $\mathrm{p}=0,011$, respectivamente). En hombres con hipertensión arterial se evidenció una concentración significativamente menor de HDL2 ( $\mathrm{p}=0,031)$, HDL3 ( $\mathrm{p}=0,028)$ y relación HDL2/HDL3 $(\mathrm{p}=0,028)$. Igualmente, estos individuos mostraron una concentración significativamente menor de HDL2 en presencia de IR $(p=0,050)$ y SM $(p=0,003)$ $\mathrm{y}$ los hombres con PCR-us elevada también mostraron una menor concentración de HDL3 ( $\mathrm{p}=0,011)$.

\section{DISCUSIÓN}

Las HDL son moléculas que pueden existir en múltiples isoformas, la heterogeneidad es una de sus principales propiedades. $\mathrm{Si}$ bien la concentración de HDL-C ha sido correlacionada inversamente con el riesgo de ECV y aterosclerosis, esta heterogeneidad le confiere efectos adicionales a sus propiedades antiaterogénicas, atribuidas al funcionalismo de las diversas moléculas que la constituyen ${ }^{(6)}$.

Durante décadas se ha planteado que las subfracciones de HDL podrían relacionarse de manera más específica con la aparición de eventos cardiovasculares; sin embargo, aunque los resultados han sido positivos no existen evidencias concluyentes acerca de cuál subclase es la más importante en este contexto ${ }^{(10,18,19)}$, esto probablemente influye en la ausencia de recomendaciones internacionales para la utilización de estos parámetros bioquímicos, de hecho ni en la guía norteamericana o europea más reciente de diagnóstico y manejo de dislipidemia se mencionan las subfracciones de HDL.

Ante este escenario controversial y la falta de reportes que evalúen las subclases de HDL en poblaciones latino- 
Tabla 3. Subfracciones de HDL según características sociodemográficas y hábitos en sujetos con HDL-C bajas

\begin{tabular}{|c|c|c|c|c|c|c|c|c|c|c|c|c|c|c|}
\hline \multirow{3}{*}{ Características } & \multicolumn{7}{|c|}{ Mujeres } & \multicolumn{7}{|c|}{ Hombres } \\
\hline & \multirow[b]{2}{*}{$\mathbf{n}$} & \multicolumn{2}{|c|}{ HDL2 } & \multicolumn{2}{|c|}{ HDL3 } & \multicolumn{2}{|c|}{ HDL2/HDL3 } & \multirow[b]{2}{*}{$\mathbf{n}$} & \multicolumn{2}{|c|}{ HDL2 } & \multicolumn{2}{|c|}{ HDL3 } & \multicolumn{2}{|c|}{ HDL2/HDL3 } \\
\hline & & $\begin{array}{l}\text { Media } \\
\text { (DE) }\end{array}$ & $\begin{array}{l}\text { Valor } \\
\text { de } p^{*}\end{array}$ & $\begin{array}{l}\text { Media } \\
\text { (DE) }\end{array}$ & $\begin{array}{l}\text { Valor } \\
\text { de } p^{*}\end{array}$ & $\begin{array}{l}\text { Media } \\
\text { (DE) }\end{array}$ & $\begin{array}{l}\text { Valor } \\
\text { de } p^{*}\end{array}$ & & $\begin{array}{l}\text { Media } \\
\text { (DE) }\end{array}$ & $\begin{array}{l}\text { Valor } \\
\text { de } p^{*}\end{array}$ & Media (DE) & $\begin{array}{l}\text { Valor } \\
\text { de } p^{*}\end{array}$ & $\begin{array}{l}\text { Media } \\
\text { (DE) }\end{array}$ & $\begin{array}{l}\text { Valor } \\
\text { de } p^{*}\end{array}$ \\
\hline \multicolumn{15}{|l|}{ Grupo etario (años) } \\
\hline$<30$ & 27 & $15,7(8,0)$ & 0,801 & $21,9(6,6)$ & 0,978 & $0,9(0,7)$ & 0,899 & 34 & $11,6(4,9)$ & 0,414 & $22,6(5,3)$ & 0,092 & $0,6(0,3)$ & 0,160 \\
\hline $30-49$ & 40 & $14,4(5,8)$ & & $21,6(6,4)$ & & $0,8(0,5)$ & & 35 & $13,1(5,3)$ & & $20,3(6,9)$ & & $0,8(0,5)$ & \\
\hline$\geq 50$ & 32 & $15,8(6,6)$ & & $21,8(7,1)$ & & $0,8(0,5)$ & & 21 & $11,1(3,9)$ & & $22,9(5,5)$ & & $0,5(0,3)$ & \\
\hline \multicolumn{15}{|l|}{ Estado civil } \\
\hline Soltero & 38 & $15,5(7,3)$ & 0,926 & $21,9(6,8)$ & 0,935 & $0,8(0,6)$ & 0,978 & 38 & $11,7(4,7)$ & 0,381 & $21,5(6,1)$ & 0,613 & $0,7(0,5)$ & 0,700 \\
\hline Casado & 38 & $14,9(6,1)$ & & $21,7(6,5)$ & & $0,8(0,5)$ & & 36 & $12,4(4,9)$ & & $22,1(6,1)$ & & $0,6(0,3)$ & \\
\hline Empleado & 44 & $15,0(6,2)$ & 0,989 & $22,4(6,7)$ & 0,453 & $0,8(0,5)$ & 0,718 & 69 & $12,4(4,9)$ & 0,235 & $21,5(5,9)$ & 0,429 & $0,7(0,4)$ & 0,235 \\
\hline Desempleado & 55 & $15,4(7,0)$ & & $21,3(6,6)$ & & $0,9(0,6)$ & & 21 & $10,9(4,6)$ & & $22,9(6,7)$ & & $0,6(0,4)$ & \\
\hline \multicolumn{15}{|l|}{ Nivel educativo } \\
\hline Hasta primaria & 23 & $16,3(5,1)$ & 0,332 & $19,0(5,8)$ & 0,020 & $0,9(0,5)$ & 0,062 & 12 & $9,9(3,9$ & 0,052 & $21,3(5,6)$ & 0,986 & $0,5(0,3)$ & 0,254 \\
\hline Secundaria & 42 & $15,7(7,8)$ & & $21,4(6,1)$ & & $0,9(0,6)$ & & 41 & $13,5(5,1)$ & & $21,9(6,7)$ & & $0,7(0,4)$ & \\
\hline Superior & 34 & $13,9(6,1)$ & & $24,2(7,1)$ & & $0,7(0,5)$ & & 37 & $11,2(4,5)$ & & $21,8(5,7)$ & & $0,6(0,4)$ & \\
\hline \multicolumn{15}{|l|}{$\begin{array}{l}\text { Estrato socioeco- } \\
\text { nómico }\end{array}$} \\
\hline I-II & 17 & $13,8(8,3)$ & 0,231 & $25,5(6,6)$ & $<0,001$ & $0,7(0,6)$ & 0,012 & 23 & $12,4(4,5)$ & 0,170 & $21,22(5,31)$ & 0,585 & $0,7(0,5)$ & 0,204 \\
\hline III & 44 & $14,9(6,6)$ & & $23,0(6,7)$ & & $0,8(0,5)$ & & 35 & $11,0(4,8)$ & & $22,69(6,27)$ & & $0,6(0,3)$ & \\
\hline Mestiza & 74 & $15,4(6,9)$ & 0,040 & $22,23(6,6)$ & 0,108 & $0,8(0,5)$ & 0,007 & 69 & $12,3(4,6)$ & 0,197 & $21,3(5,8)$ & 0,156 & $0,7(0,4)$ & 0,130 \\
\hline Blanco hispánica & 10 & $9,8(4,2)$ & & $23,5(7,5)$ & & $0,5(0,3)$ & & 16 & $11,9(6,1)$ & & $24,0(6,8)$ & & $0,6(0,4)$ & \\
\hline Afrovenezolana & 1 & - & & - & & - & & 2 & $8,4(8,5)$ & & $28,4(4,4)$ & & $0,3(0,4)$ & \\
\hline $\begin{array}{l}\text { Indígena } \\
\text { americano }\end{array}$ & 3 & $17,8(5,0)$ & & $18,1(4,7)$ & & $1,1(0,5)$ & & 3 & $10,2(1,8$ & & $17,8(2,7)$ & & $0,6(0,1)$ & \\
\hline \multicolumn{15}{|l|}{ Consumo de alcohol } \\
\hline Sí & 13 & $12,7(6,4)$ & 0,125 & $24,2(7,0)$ & 0,171 & $0,6(0,5)$ & 0,082 & 47 & $12,1(4,9)$ & 0,904 & $22,0(6,0)$ & 0,614 & $0,61(0,32)$ & 0,897 \\
\hline No & 86 & $15,6(6,7)$ & & $21,4(6,5)$ & & $0,9(0,5)$ & & 43 & $12,1(4,8)$ & & $21,5(6,2)$ & & $0,67(0,46)$ & \\
\hline \multicolumn{15}{|l|}{ Hábito tabáquico } \\
\hline No & 80 & $14,9(6,6)$ & 0,496 & $22,4(6,6)$ & 0,034 & $0,8(0,6)$ & 0,124 & 61 & $12,1(5,3)$ & 0,916 & $22,0(6,5)$ & 0,735 & $0,65(0,43)$ & 0,837 \\
\hline Fumador & 11 & $14,9(6,6)$ & & $20,5(4,9)$ & & $0,8(0,4)$ & & 12 & $12,2(4,2)$ & & $20,2(4,7)$ & & $0,64(0,25)$ & \\
\hline Exfumador & 8 & $17,2(4,6)$ & & $17,1(7,6)$ & & $1,1(0,5)$ & & 17 & $11,8(3,8)$ & & $22,1(5,4)$ & & $0,60(0,34)$ & \\
\hline \multicolumn{15}{|l|}{ AF en esfera ocio } \\
\hline Ninguna & 67 & $15,0(6,3)$ & 0,245 & $21,1(5,9)$ & 0,571 & $0,8(0,5)$ & 0,326 & 45 & $11,6(4,7)$ & 0,142 & $22,5(6,2)$ & 0,077 & $0,6(0,4)$ & 0,038 \\
\hline Muy baja & 3 & $7,5(1,1)$ & & $23,9(6,9)$ & & $0,3(0,2)$ & & 11 & $14,2(5,5)$ & & $19,2(6,8)$ & & $0,8(0,4)$ & \\
\hline Baja & 12 & $16,4(6,0)$ & & $22,5(7,2)$ & & $0,9(0,6)$ & & 6 & $13,2(4,6)$ & & $19,2(4,9)$ & & $0,7(0,3)$ & \\
\hline Moderada & 8 & $14,5(8,1)$ & & $26,7(9,9)$ & & $0,8(0,8)$ & & 9 & $9,8(5,9)$ & & $26,7(6,0)$ & & $0,4(0,3)$ & \\
\hline Alta & 3 & $17,3(6,8)$ & & $22,1(6,5)$ & & $0,91(0,7)$ & & 8 & $10,9(3,3)$ & & $21,0(3,1)$ & & $0,5(0,2)$ & \\
\hline Muy alta & 6 & $18,9(10,3)$ & & $19,6(6,8)$ & & $1,2(0,9)$ & & 11 & $13,9(4,0)$ & & $19,7(4,8)$ & & $0,8(0,4)$ & \\
\hline
\end{tabular}

AF: actividad física; DE: desviación estándar.

${ }^{\varsigma}$ Consumidor $>1$ g/día.

*Prueba t-Student para comparar entre dos categorías o prueba ANOVA de un factor para comparar entre tres o más categorías.

En pacientes con HDL normales no se observaron diferencias significativas entre las medias de los grupos. 
Tabla 4. Subfracciones de HDL según características clínico-metabólicas y sexo de sujetos con HDL-C bajas.

\begin{tabular}{|c|c|c|c|c|c|c|c|}
\hline \multirow{2}{*}{ Característica } & \multicolumn{3}{|c|}{ HDL 2} & \multirow{2}{*}{$\begin{array}{c}\text { HDL } 3 \\
\text { Media (DE) }\end{array}$} & \multicolumn{3}{|c|}{ HDL2/HDL3 } \\
\hline & $\mathbf{n}$ & Media (DE) & Valor de $\mathbf{p}^{*}$ & & Valor de $\mathbf{p}^{*}$ & Media (DE) & Valor de $\mathbf{p}^{*}$ \\
\hline \multicolumn{8}{|l|}{ Mujeres } \\
\hline \multicolumn{8}{|l|}{$\mathrm{TAG}(\mathrm{mg} / \mathrm{dL})^{\dagger}$} \\
\hline$<150$ & 75 & $16,2(6,9)$ & 0,033 & $22,5(6,5)$ & 0,034 & $0,8(0,6)$ & 0,608 \\
\hline$\geq 150$ & 24 & $12,3(4,8)$ & & $19,4(6,4)$ & & $0,7(0,5)$ & \\
\hline \multicolumn{8}{|l|}{$\operatorname{IMC}\left(\mathrm{kg} / \mathrm{m}^{2}\right)$} \\
\hline$\leq 24,9$ & 27 & $15,5(7,9)$ & 0,976 & $23,2(6,0)$ & 0,041 & $0,8(0,6)$ & 0,516 \\
\hline $25-29,9$ & 32 & $15,0(5,8)$ & & $22,9(6,5)$ & & $0,8(0,5)$ & \\
\hline$\geq 30$ & 40 & $15,2(6,6)$ & & $19,9(6,8)$ & & $0,9(0,5)$ & \\
\hline \multicolumn{8}{|l|}{$\mathrm{CABD}^{\dagger}$} \\
\hline Normal & 18 & $14,7(8,4)$ & 0,448 & $23,7(5,5)$ & 0,110 & $0,7(0,6)$ & 0,203 \\
\hline Elevada & 81 & $15,3(6,3)$ & & $21,4(6,8)$ & & $0,8(0,5)$ & \\
\hline \multicolumn{8}{|l|}{ Clasificación de PA } \\
\hline Normotensos & 36 & $15,3(7,0)$ & 0,186 & $22,5(6,1)$ & 0,237 & $0,8(0,6)$ & 0,116 \\
\hline HTA 1 & 16 & $14,2(6,1)$ & & $22,7(7,8)$ & & $0,7(0,4)$ & \\
\hline HTA 2 & 7 & $20,5(4,8)$ & & $17,2(5,0)$ & & $1,3(0,6)$ & \\
\hline \multicolumn{8}{|l|}{$\mathrm{IR}^{\prime}$} \\
\hline Ausente & 55 & $16,2(6,7)$ & 0,090 & $21,5(6,0)$ & 0,773 & $0,9(0,5)$ & 0,181 \\
\hline Presente & 44 & $13,9(6,5)$ & & $22,2(7,3)$ & & $0,8(0,6)$ & \\
\hline \multicolumn{8}{|l|}{ PCR-us (mg/L) } \\
\hline$<0,765$ & 38 & $14,2(7,3)$ & 0,342 & $25,8(6,0)$ & $<0,001$ & $0,6(0,5)$ & 0,011 \\
\hline$\geq 0,765$ & 25 & $15,3(6,4)$ & & $19,1(5,0)$ & & $0,9(0,5)$ & \\
\hline \multicolumn{8}{|l|}{$\operatorname{Lp}(\mathrm{a})$} \\
\hline$<30$ & 54 & $15,3(6,9)$ & 0,956 & $22,9(7,2)$ & 0,128 & $0,8(0,6)$ & 0,499 \\
\hline$\geq 30$ & 43 & $14,9(6,2)$ & & $20,5(5,7)$ & & $0,8(0,5)$ & \\
\hline \multicolumn{8}{|l|}{$\mathrm{SM}^{+}$} \\
\hline Ausente & 49 & $16,2(6,9)$ & 0,190 & $21,9(6,2)$ & 0,634 & $0,9(0,6)$ & 0,481 \\
\hline Presente & 50 & $14,2(6,3)$ & & $21,6(7,0)$ & & $0,8(0,5)$ & \\
\hline$<150$ & 49 & $12,8(5,0)$ & 0,188 & $21,9(5,9)$ & 0,712 & $0,7(0,4)$ & 0,450 \\
\hline$\geq 150$ & 41 & $11,3(4,6)$ & & $21,6(16,2)$ & & $0,6(0,4)$ & \\
\hline \multicolumn{8}{|l|}{$\operatorname{IMC}\left(\mathrm{kg} / \mathrm{m}^{2}\right)$} \\
\hline$\leq 24,9$ & 15 & $13,4(3,9)$ & 0,334 & $19,8(4,7)$ & 0,230 & $0,8(0,4)$ & 0,308 \\
\hline $25-29,9$ & 37 & $12,2(4,9)$ & & $23,0(6,3)$ & & $0,6(0,3)$ & \\
\hline$\geq 30$ & 38 & $11,5(5,1)$ & & $21,4(6,2)$ & & $0,6(0,5)$ & \\
\hline $\mathrm{CABD}^{+}$ & & & & & & & \\
\hline Normal & 19 & $13,5(4,1)$ & 0,119 & $20,1(4,8)$ & 0,261 & $0,7(0,4)$ & 0,110 \\
\hline Elevada & 71 & $11,7(4,9)$ & & $22,2(6,3)$ & & $0,6(0,4)$ & \\
\hline Clasificación de PA & & & & & & & \\
\hline Normotensos & 33 & $13,0(4,4)$ & 0,031 & $20,7(5,4)$ & 0,028 & $0,7(0,4)$ & 0,028 \\
\hline Prehipertensos & 32 & $11,4(4,3)$ & & $20,4(5,8)$ & & $0,7(0,5)$ & \\
\hline HTA 1 & 18 & $12,9(5,8)$ & & $24,4(7,1)$ & & $0,6(0,3)$ & \\
\hline HTA 2 & 7 & $8,3(4,9)$ & & $26,5(3,3)$ & & $0,3(0,2)$ & \\
\hline $\mathrm{IR}^{\prime}$ & & & & & & & \\
\hline Ausente & 45 & $12,8(4,2)$ & 0,050 & $21,7(5,9)$ & 0,991 & $0,7(0,3)$ & 0,171 \\
\hline Presente & 45 & $11,3(5,3)$ & & $21,9(6,3)$ & & $0,6(0,4)$ & \\
\hline PCR-us (mg/L) & & & & & & & \\
\hline$<0,765$ & 58 & $11,2(5,2)$ & 0,472 & $23,2(6,3)$ & 0,011 & $0,6(0,4)$ & 0,103 \\
\hline$\geq 0,765$ & 19 & $11,9(4,0)$ & & $18,9(4,9)$ & & $0,7(0,5)$ & \\
\hline $\operatorname{Lp}(\mathrm{a})$ & & & & & & & \\
\hline$<30$ & 44 & $10,7(5,0)$ & 0,082 & $22,9(4,9)$ & 0,167 & $0,5(0,3)$ & 0,064 \\
\hline$\geq 30$ & 31 & $12,8(4,7)$ & & $21,5(7,7)$ & & $0,7(0,5)$ & \\
\hline $\mathrm{SM}^{+}$ & & & & & & & \\
\hline Ausente & 31 & $13,7(3,9)$ & 0,003 & $20,1(4,8)$ & 0,122 & $0,8(0,4)$ & 0,011 \\
\hline Presente & 59 & $11,2(5,1)$ & & $22,7(6,5)$ & & $0,6(0,4)$ & \\
\hline
\end{tabular}

TAG: triacilglicéridos; IMC: índice de masa corporal; CABD: circunferencia abdominal; HTA: hipertensión arterial; PA: presión arterial; IR: insulinorresistencia; PCR-us: proteína C reactiva ultrasensile; SM: síndrome metabólico.

${ }^{\dagger}$ Criterios según consenso IDF/NHLBI/AHA; ' Criterios según EPSMM (HOMA2-IR $\geq 2$ ).

* Prueba t-Student para comparar entre dos categorías o prueba ANOVA de un factor para comparar entre tres o más categorías.

En pacientes con HDL normales no se observaron diferencias significativas entre las medias de los grupos. 
americanas, especialmente en Venezuela, el presente estudio describe las principales características clínicas y epidemiológicas de las subfracciones de HDL en la población de la ciudad de Maracaibo, la segunda ciudad más importante de Venezuela en términos poblacionales y económicos. En efecto, para el periodo de recolección de la muestra, Maracaibo presentaba una alta frecuencia de factores de riesgo cardiovascular y de hábitos psicobiológicos deletéreos como sedentarismo y consumo alto de grasas saturadas ${ }^{(20,21)}$.

En todo análisis epidemiológico de una medición de laboratorio cuyos reportes son escasos en una región o país es importante mostrar sus valores promedio y medidas de dispersión. $\mathrm{Al}$ comparar los resultados en las subfracciones de HDL-C obtenidas en este estudio con respecto a otras poblaciones encontramos cifras que difieren, así por ejemplo, Kim et al. ${ }^{(22)}$ encontró en una población europea un promedio de HDL3 más alto (mujeres: $49 \pm 11 \mathrm{mg} / \mathrm{dL}$ y hombres: $39 \pm 11 \mathrm{mg} / \mathrm{dL}$ ), mientras que el promedio de HDL2 fue más elevado en mujeres (mujeres: $14,7 \pm 7,8 \mathrm{mg} / \mathrm{dL}$ y hombres: $8,5 \pm 5,3 \mathrm{mg} / \mathrm{dL}$ ). Por su parte, en un estudio realizado por Koumaré et al. ${ }^{(23)}$, en sujetos de Burkina Faso el promedio de HDL3 fue igual en hombres y mujeres (26,2 mg/dL), y el promedio de HDL2 en hombres fue 15,4 mg/ $\mathrm{dL}$ y en mujeres fue $18,9 \mathrm{mg} / \mathrm{dL}$, valores similares a los obtenidos en esta investigación.

Es importante mencionar que, en general, se observan diferencias en los promedios de las subfracciones de HDL en los sujetos con HDL-C bajas. En relación con los datos sociodemográficos, los indígenas americanos presentaron niveles más elevados de HDL2 y HDL2/HDL3 al compararse con individuos blanco-hispánicos, datos que difieren con el estudio llevado a cabo por Martin et al. ${ }^{(24)}$, en donde no se evidenció relación entre los grupos étnicos y las diferentes subfracciones de HDL-C.

En cuanto a las características clínico-metabólicas, se ha descrito que las dislipidemias representan un factor de riesgo cardiovascular frecuente y que coexisten frecuentemente los niveles elevados de triacilglicéridos con HDL-C bajas y alteraciones en la distribución de sus subfracciones ${ }^{(25)}$. Nuestros hallazgos muestran que en las mujeres con hipertriacilgliceridemia los niveles de ambas subfracciones eran significativamente menores, lo cual se diferencia de los resultados de Jia et al. ${ }^{(26)}$, quienes observaron que los sujetos hiperlipémicos presentaban niveles más bajos de HDL2 pero más elevados de HDL3 que en los normolipémicos. De igual forma, Gou et al. ${ }^{(27)}$ encontraron que en las mujeres con hipertriacilgliceridemia, los niveles de HDL2 eran significantivamente menores que en los eulipémicos, mientras que el promedio de HDL3 era mayor en este último grupo. Estas diferencias podrían explicarse por el método de obtención de las subfracciones de HDL-C y por el tamaño de la muestra; no obstante, estos resultados sugieren una relación inversamente proporcional entre la hipertriacilgliceridemia y las HDL2.
Asimismo, los niveles bajos de HDL-C han sido asociados a inflamación de bajo grado, IR y obesidad visceral. En este sentido, los resultados obtenidos muestran niveles de HDL3 disminuidos en sujetos con PCR-us elevada en ambos sexos, mientras que aquellos con SM y presencia de IR exhibieron niveles más bajos de HDL2 en hombres. Estos resultados coinciden con los hallazgos del estudio ELSA-Brasil, uno de los pocos reportes latinoamericanos que exhibe características de las subclases de HDL-C en nuestra región, en el cual tanto la concentración de HDL-C como sus subfracciones se asociaron inversamente con inflamación de bajo grado, IR y $\mathrm{SM}^{(27)}$. De forma similar, otro estudio brasileño que evaluó el impacto de la IR en el metabolismo de diferentes lipoproteínas demostró porcentajes más bajos de las subfracciones más grandes (HDL2 y HDL3) en los sujetos con IR ${ }^{(28)}$, estos hallazgos evidencian el papel que mediaría la estructura y función de las HDL-C como lipoproteínas cardioprotectoras.

Los mecanismos moleculares implicados en la relación inversa entre subfracciones de HDL-C y mediadores inflamatorios, como la PCR-us, aún no han sido precisados. No obstante, los menores niveles de HDL3 en sujetos con PCR-us elevada podría interpretarse como una menor cantidad de lipoproteínas nacientes disponibles para movilizar y externalizar el colesterol desde las células espumosas endoteliales, lo cual conllevaría a la activación y acumulación de los desencadenantes del fenómeno inflamatorio. Otro reporte latinoamericano, muestra niveles más bajos de HDL2 y HDL3 específicamente en sujetos diabéticos peruanos ${ }^{(29)}$, asociación que no fue significativa en nuestro estudio.

Respecto a la razón HDL2/HDL3, los valores observados en este estudio solo mostraron relación con el estatus socioeconómico, raza y PCR-us para las muejres; y relación con la actividad física, presión arterial y SM para los hombres. En este sentido, Moriyama et al. ${ }^{(30)}$ han llevado a cabo varias investigaciones en la población japonesa y han evidenciado que los cambios en la razón HDL2/HDL3 se correlacionan inversamente con la circunferencia abdominal e IR, y positivamente con hábitos de vida saludables, por lo que es utilizado como un marcador útil para SM y condiciones aterogénicas en esa población.

Entre las limitaciones del estudio se pueden mencionar: el tamaño muestral impide generalizar los resultados a toda la población, el diseño transversal imposibilita establecer relaciones de causalidad ni de recomendación de uso clínico de las subfracciones de HDL-C y los cambios socioeconómicos que afectan a la población de estudio durante los últimos años podrían influir en los resultados mostrados.

En conclusión, las subfracciones de HDL-C tienen un comportamiento clínico epidemiológico variable en individuos adultos de la población de Maracaibo, con promedios más bajos en los hombres, diferencias en los niveles socioeconómicos únicamente en aquellos con HDL-C bajas, y sin predominio 
de alguna subclase según las características sociodemográficas, clínicas y bioquímicas. Por lo cual, se sugiere profundizar el estudio de estas lipoproteínas en la población latinoamericana, haciendo énfasis en su relación con distintos trastornos que conllevan a un mayor riesgo cardiometabólico.

Contribuciones de los autores: SL y VB concibieron y diseñaron el estudio. JS, JR, MC y MM realizaron los análisis. MN. LO, AO AM y MC contribuyeron a la adquisición de datos, su interpretación y a la redacción del manuscrito. Todos los autores contribuyeron a la discusión, revisión, aprobación del manuscrito final y asumen responsabilidad de sus contenidos.

Fuentes de financiamiento: El presente estudio fue financiado por el Consejo de Desarrollo Científico, Humanístico y Tecnológico CONDES (CC-0437-10-21-09-10) y por Fundacite-Zulia (FZ-00582007).

Conflictos de interés: Los autores no tienen ningún conflicto de interés que declarar.

\section{REFERENCIAS BIBLIOGRÁFICAS}

1. Besler C, Lüscher T, Landmesser U. Molecular mechanisms of vascular effects of High-density lipoprotein: alterations in cardiovascular disease. EMBO Molecular Medicine. 2012;4(4):251-268. doi: 10.1002/ emmm.201200224.

2. GBD 2017 Causes of Death Collaborators. Global, regional, and national age-sex-specific mortality for 282 causes of death in 195 countries and territories, 1980-2017: a systematic analysis for the Global Burden of Disease Study 2017. Lancet. 2018;392:1736-88. doi: 10.1016/S01406736(18)32203-7.

3. Bermudez V, Salazar J, Rojas J, Martínez MS, Bello L, Añez R, et al. Prevalence, Lipid Abnormalities Combinations and Risk Factors Associated with Low HDL-C Levels in Maracaibo City, Venezuela. J J Commun Med. 2015;1(2):009.

4. Bermúdez V, Salazar J, Bello L, Rojas J, Añez R, Roque W, et al. Coronary Risk Estimation According to a Recalibrated Framingham-Wilson Score in the Maracaibo City Metabolic Syndrome Prevalence Study. The Journal for Cardiology. Photon 2014;107:160-170.

5. Salazar J, Cabrera M, Ramos E, Olivar L, Aguirre M, Rojas J, et al. HDL-C y riesgo de aterosclerosis. Diabetes Internacional. 2013;5(2):42-54.

6. Salazar J, Olivar LC, Ramos E, Chávez-Castillo M, Rojas J, Bermudez V.Dysfunctional High-Density Lipoprotein: An Innovative Target for Proteomics and Lipidomics. Cholesterol. 2015;2015:296417. doi: 10.1155/2015/296417.

7. Martin S, Jones S, Toth P. High-density lipoprotein subfractions: current views and clinical practice applications. Trends Endocrinol Metab. 2014;25(7):329-36. doi: 10.1016/j.tem.2014.05.005.

8. De Lalla OF, Gofman JW. Ultracentrifugal analysis of serum lipoproteins. Methods Biochem Anal.1954;1:459-478. doi: 10.1002/9780470110171.ch16.

9. Pirillo A, Norata G, Catapano A. High-Density Lipoprotein Subfractions - What the Clinicians Need to Know. Cardiology. 2013;124(2):116-125. doi: 10.1159/000346463.

10. Davidson M. The Battle of the HDL Subfractions. Cardiovasc Revasc Med. 2019;20(11):943-944. doi: 10.1016/j.carrev.2019.09.013.

11. Bermúdez V, Marcano R, Cano C, Arráiz N, Amell A, Cabrera M, et al. The Maracaibo City Metabolic Syndrome Prevalence Study: Design and Scope. Am J Ther. 2010;17(3):288-294. doi: 10.1097/MJT.0b013e3181c121bc.

12. Chobanian AV, Bakris GL, Black HR, Cushman WC, Green LA, Izzo JL $\mathrm{Jr}$, et al. Joint National Committee on Prevention, Detection, Evaluation, and Treatment of High Blood Pressure. The seventh report of the joint national committee on prevention, detection, evaluation, and treatment of high blood pressure. JAMA. 2003;289:2560-2571. doi: 10.1161/01. HYP.0000107251.49515.c2.

13. World Health Organization. Obesity: preventing and managing the global epidemic. Report of a WHO consultation. World Health Organ Tech Rep Ser [Internet]. WHO; 2000 [citado el 01 de agosto de 2019]. Disponible en: https://www.who.int/nutrition/publications/obesity/ WHO_TRS_894/en/.

14. Alberti K, Eckecl R, Grundy S, Zimmer PZ, Cleeman JI, Donato KA, et al. Harmonizing the Metabolic Syndrome: A Joint Interim Statement of the International Diabetes Federation Task Force on Epidemiology and
Prevention: National Heart, Lung, and Blood Institute; American Heart Association; World Heart Federation; International Atherosclerosis Society; International Association for the Study of Obesity. Circulation. 2009;120:1640-45. doi: 10.1161/CIRCULATIONAHA.109.192644.

15. Bermúdez V, Cabrera M, Mendoza L, Chávez M, Martínez M, Rojas J, et al. High-sensitivity c-reactive protein epidemiological behavior in adult individuals from Maracaibo, Venezuela. Revista Latinoamericana de Hipertensión. 2013;8(1):22-29.

16. Bermúdez V, Rojas J, Salazar J, Bello L, Áñez R, Toledo A, et al. Variations of Lipoprotein(a) Levels in the Metabolic Syndrome: A Report from the Maracaibo City Metabolic Syndrome Prevalence Study. J Diabetes Res. 2013;2013:416451. doi: 10.1155/2013/416451.

17. Bermúdez V, Rojas J, Martínez M, Apruzzese V, Chávez-Castillo M, Gonzalez R, et al. Epidemiologic Behavior and Estimation of an Optimal Cut-Off Point for Homeostasis Model Assessment-2 Insulin Resistance: A Report from a Venezuelan Population. Int Sch Res Notices. 2014;2014:616271. doi: 10.1155/2014/616271.

18. Williams P. Fifty-three year follow-up of coronary heart disease versus HDL2 and other lipoproteins in Gofman's Livermore Cohort. J Lipid Res. 2012;53(2):266-272. doi: 10.1194/jlr.M019356.

19. Chaudhary R, Kinderytė M, Chaudhary R, Sukhi A, Bliden K, Tantry $\mathrm{U}$, et al. HDL3-C is A Marker of Coronary Artery Disease Severity and Inflammation in Patients on Statin Therapy. Cardiovasc Revasc Med. 2019;20:1001-6. doi: 10.1016/j.carrev.2018.12.019.

20. Bermudez V, Rojas J, Salazar J, Calvo MJ, Morillo J, Torres W, et al. The Maracaibo city metabolic syndrome prevalence study: primary results and agreement level of 3 diagnostic criteria. Revista Latinoamericana de Hipertensión. 2014;9(4):20-31.

21. Souki A, Sandoval M, Sánchez G, Andrade U, García-Rondón D, Cano $\mathrm{C}$, et al. Intake of saturated fatty acids and sensitivity to insulin in obese young adults in Maracaibo. Revista Latinoamericana de Hipertensión. 2007;3(5):159-165.

22. Kim DS, Burt AA, Rosenthal EA, Ranchalis JE, Eintracht JF, Hatsukami TS, et al. HDL-3 is a superior predictor of carotid artery disease in a case-control cohort of 1725 participants. J Am Heart Assoc. 2014;3(3):e000902. doi: 10.1161/JAHA.114.000902.

23. Koumaré AT, Sakandé LP, Kabré E, Sondé I, Simporé J, Sakandé J. Reference ranges of cholesterol sub-fractions in random healthy adults in Ouagadougou, Burkina Faso. PLoS One. 2015;10(1):e011642. doi: 10.1371/journal.pone.0116420.

24. Martin SS, Khokhar AA, May HT, Kulkarni KR, Blaha MJ, Joshi PH, et al. HDL cholesterol subclasses, myocardial infarction, and mortality in secondary prevention: the Lipoprotein Investigators Collaborative. Eur Heart J. 2015;36(1):22-30. doi: 10.1093/eurheartj/ehu264.

25. Quintanilla-Cantú A, Peña-de-la-Sancha P, Flores-Castillo C, Mejía-Domínguez AM, Posadas-Sánchez R, Pérez-Hernández N, et al.Small HDI 
subclasses become cholesterol-poor during postprandial period after a fat diet intake in subjects with high triglyceridemia increases. Clin Chim Acta. 2017;464:98-105. doi: 10.1016/j.cca.2016.11.018.

26. Jia L, Fu M, Tian Y, Xu Y, Gou L, Tian H, et al. Alterations of high-density lipoprotein subclasses in hypercholesterolemia and combined hyperlipidemia. Int J Cardiol. 2007;120(3):331-7. doi: 10.1016/j.ijcard.2006.10.007.

27. Generoso G, Bensenor IM, Santos RD, Santos IS, Goulart AC, Jones SR, et al. Association between high-density lipoprotein subfractions and low-grade inflammation, insulin resistance, and metabolic syndrome components: The ELSA-Brasil study. J Clin Lipidol. 2018;12(5):12901297. doi: 10.1016/j.jacl.2018.05.003.

28. Cartolano FDC, Dias GD, Freitas MCP de, Figueiredo Neto AM, Damasceno NRT. Insulin Resistance Predicts Atherogenic Lipoprotein Profile in Nondiabetic Subjects.J Diabetes Res. 2017;2017:1018796. doi: 10.1155/2017/1018796.

29. Jo N, Garmendia F, Pando R. Sub-clases de lipoproteínas de alta densidad en diabéticos. Rev méd peru.1994;66(351):68-70.

30. Moriyama K, Takahashi E. HDL2/HDL3 ratio changes, metabolic syndrome markers, and other factors in a Japanese population. J Atheroscler Thromb. 2016;23(6):704-712. doi: 10.5551/jat.32896. 